\title{
Morteros ligeros con aditivos químicos y zeolitas naturales sílico-activas*
}

REGINO SOTOLONGO LORENTE, REGINO GAYOSO BLANCO Y CARLOS GIL IZQUIERDO Dpto. de Hormigón Hidráulico. Centro Técnico para el desarrollo de los Materiales de Construcción

Fecha de recepción: 10-V-94.

CUBA

RESUMEN

Se evalúa la influencia de aditivos químicos (superplastificante-retardador) y minerales silico-activos (fracción menor que $45 \mu \mathrm{m}$ ) en la hidratación del cemento, incremento de resistencia mecánica y variaciones en las propiedades fisicas de morteros ligeros con tobas cubanas parcialmente zeolitizadas.
SUMMARY

The influence of chemical admixtures (superplasticizerretarder) and active silica minerals (fraction less than 45 $\mu \mathrm{m})$ in cement hydration, mechanical strength increase and variations of the physical propierties of light weigth mortars with partially zeolitized Cuban tuffs is evaluated.

\section{INTRODUCCIÓN}

Las posibilidades de utilización de las tobas parcialmente zeolitizadas, comoáridos en morteros y hormigones ligeros de alta resistencia, han estado en gran medida limitadas por la alta porosidad, capacidad y velocidad de absorción de agua característica de su estructura mineral.

Los progresos alcanzados en la tecnología del hormigón con el empleo, cada vez más creciente de los aditivos químicos, ha permitido en la actualidad aprovechar las ventajas del empleo de las tobas comoárido ligero natural, posibilitando la sustitución de áridos silíceos cuarzosos convencionales, de mayor densidad y limitadas reservas en Cuba.

En este sentido han jugado un roll importante las formulaciones y experiencias de empleo en morteros para barcos de ferrocemento, en la industria pesquera cubana, que incluye el uso de aditivos superplastificantes para atenuar los excesos de agua de amasado en las mezclas de alta consistencia y sacarosa como regulador del fraguado, evitando pérdidas acentuadas en la laborabilidad durante los primeros 45 minutos.

A partir de los contenidos de aditivos establecidos, para cumplir los requisitos tecnológicos citados, en este trabajo se estudia la hidratación del cemento portland y su influencia sobre las propiedades físico-mecánicas de pastas y morteros en el tiempo.

\section{MATERIALES EMPLEADOS}

-Cemento P 35.- Fábrica Carlos Marx. Cienfuegos, (tabla 1).

-Árido de tobas.- Yacimiento de Tasajeras. Las Villas. Tipo litológico I y III. Fracciones 0-45 $\mu \mathrm{m}$ y 0-2,38 mm. -Aditivos químicos.- Superplastificante (A.S.P.) de resina de formaldehído condensado naftaleno sulfonado.

Retardador de fraguado (R.F.) sacarosa.

(*) Continuación del trabajo: " Contribución al estudio de la sacarosa como aditivo retardador de la hidratación del cemento " Ref./2/. 
TABLA 1

CARACTERISTICAS DEL CEMENTO

\begin{tabular}{|c|c|c|c|c|c|c|c|}
\hline \multicolumn{4}{|c|}{ Composición mineralógica (Bogue) } & \multirow{2}{*}{$\begin{array}{c}\text { Superficie } \\
\text { espec/fica } \\
\left(\mathrm{cm}^{2} / \mathrm{g}\right)\end{array}$} & \multirow{2}{*}{$\begin{array}{c}\text { Masa } \\
\text { especifica } \\
\left(\mathrm{g} / \mathrm{cm}^{3}\right)\end{array}$} & \multicolumn{2}{|c|}{ Resistencia mecánica (28d)* } \\
\hline C3S & $\mathrm{C2S}$ & C3A & C4AF & & & $\begin{array}{c}\text { Flexión } \\
\text { (MPa) }\end{array}$ & $\begin{array}{c}\text { Compresión } \\
\text { (MPa) }\end{array}$ \\
\hline 54,3 & 19,2 & 4,7 & 15,2 & $2.294,7$ & 3,16 & 7,0 & 37,0 \\
\hline
\end{tabular}

* Según norma ISO.

\section{PROCEDIMIENTO EXPERIMENTAL}

Pastas de cemento.- Con la relación de materiales (tabla 2) se prepararon pastas de cemento en una mezcladora con giro planetario. Se conformaron cubos de $20 \times 20 \times 20 \mathrm{~mm}$ aplicando la vibrocompactación y se curaron bajo agua hasta la edad de ensayo. Se determinaron las propiedades reológicas por el método del minicono /1/. En la serie S5.A, se consideró el efecto reductor de agua del A.S.P., y la relación $\mathrm{A} / \mathrm{C}$ se ajustó a 0,29 . El estudio cinético de la hidratación de la alita se realizó siguiendo la metodología descrita en $/ 2 /$. El análisis de la porosidad por intrusión de mercurio en las pastas de cemento con aditivo mineral, A.S.P. y la mezcla de ambos con el A.R. se ajustó al procedimiento utilizado en $/ 3 /$.

TABLA 2

RELACIÓN DE MATERIALES Y REOLOGÍA. PASTAS DE CEMENTO

\begin{tabular}{|c|c|c|c|c|c|c|}
\hline \multirow{2}{*}{ Muestra } & \multicolumn{3}{|c|}{ \% Aditivo/ P. de cemento. } & \multirow{2}{*}{$\begin{array}{c}\text { Relación } \\
\text { A/C }\end{array}$} & \multicolumn{2}{|c|}{ Reología minicono. } \\
\hline & Toba & Sacarosa & A.S.P. & & Area $\left(\mathrm{cm}^{2}\right)$ & $\begin{array}{c}\% \text { Area } \\
\text { respecto a S. }\end{array}$ \\
\hline s & -..-. & ---- & ---- & 0,35 & 24,8 & 100 \\
\hline $\mathrm{s} 1.1$ & ---- & 0,025 & ----- & 0,35 & 25,3 & 102 \\
\hline S1.2 & -..-.- & 0,100 & ..--.- & 0,35 & 27,9 & 112 \\
\hline S1.3 & ----. & 0,200 & ----- & 0,35 & 35,4 & 143 \\
\hline 51.4 & $-\cdots$ & 0,300 & ----- & 0,35 & 45,8 & 185 \\
\hline S1.5 & -..--. & 0,400 & -..-. & 0,35 & 53,9 & 217 \\
\hline 52 & 16 & 0,400 & $\cdots$ & 0,35 & $-\cdots$ & ---- \\
\hline 53 & $\cdots$ & 0,400 & 0,600 & 0,35 & 145,8 & 585 \\
\hline s4 & 16 & 0,400 & 0,600 & 0,35 & 48,3 & 195 \\
\hline s5 & ---- & -... & 0,600 & 0,35 & 117,3 & 473 \\
\hline S5.A & ---- & ---- & 0,600 & 0,29 & 25,2 & 102 \\
\hline s6 & 16 & ---- & $-\cdots$ & 0,35 & $-\infty$ & ---- \\
\hline s7 & 16 & --.-- & 0,600 & 0,35 & 35,6 & 144 \\
\hline
\end{tabular}

Mortero.-Se prepararon mezclas de morteros con la relación de materiales de la tabla 6 y metodología de elaboración descrita en la tabla 5. En /4/ se demostró que la preparación primaria de la pasta de cemento, y la adición posterior de los áridos secos, incrementaba las propiedades mecánicas del mortero. Se conformaron prismas de $40 \times 40 \times 160 \mathrm{~mm}$ curados por inmersión en agua. Los ensayos de permeabilidad ( $\%$ de agua pasada) y absorción de agua se ajustaron a la metodología de las normas japonesas JS A.6203 y 11404 respectivamente, en ambos casos con la variante de curado contínuo por inmersión hasta la edad de ensayo.

\section{ANÁLISIS Y DISCUSIÓN DE LOS RESULTADOS}

La adición de sacarosa en $0,4 \% \mathrm{p} / \mathrm{p}$. de cemento evita pérdidas de laborabilidad a los 45 minutos superiores al $20 \%$, y no afecta la hidratación del cemento en el mortero ni las propiedades mecánicas a largo plazo(Figs. 2, 3 y 5). Los resultados reportados en $/ 2 /$, y reproducidos parcialmente en la tabla 3, confirmaron que además del contenido de sacarosa, su efecto en el retardo de la hidratación del cemento dependía de la superficie total del 
sólido en contacto con el agua de amasado (superficie específica del cemento, acción dispersante del A.S.P. y presenciade adiciones minerales con granulometría similar al cemento). Además, incrementa la laboriosidad de la mezcla y acentúa el efecto de los A.S.P. (ver reología en tabla 2).

Las tobas empleadas como áridos son rocas vitroclásticas alteradas con una composición química fundamentalmente silícea $(66,4 \%)$. Según $/ 5 /$, tienen como minerales principales la heulandita, clinoptilolita, mordenita y cuarzo con pequeñas cantidades de montmorillonita, calcita y mullita. Contiene un $10 \%$ de partículas con diámetro inferior a $45 \mu \mathrm{m}$, que actuarán como adición mineral activa. Su actividad puzolánica, probadaen/6/, se confirmó en los estudios cinéticos en pastas de cemento, al incrementar el grado de reacción $\alpha$ y la constante de velocidad de reacción $\mathrm{K}$ (tabla 3 ). Efectos similares para otras puzolanas se recogen en /7/. El contenido de poros grandes y dañinos $\left(>1.000 \mathrm{~A}^{\circ}\right)$, en las pastas con puzolana, fue inferior al patrón (tabla 4).

Al considerar el efecto reductor de agua del A.S.P. en S5.A, se observóuna disminución apreciable de la porosidad de la pasta con respecto a S5. (tabla 4), sobre todo en los poros mayores de $1.000 \mathrm{~A}^{\circ}$, que fueron a su vez inferiores al patrón. Aunque en S7. se trabajó a relación A/C fija, la absorción de agua por la toba (ver reología en tabla 2) y su efecto en la atenuación de la exudación externa /8/, disminuyó la porosidad total con respecto a S5. En S4, por el efecto retardante de la sacarosa, se obtuvo el menor refinamiento de la porosidad superior a $1.000 \mathrm{~A}^{\circ}$. No obstante, la porosidad total fue inferior a S5. y S7. Todo lo anterior demuestra que aunque el A.S.P. retardó

TABLA 3

ESTUDIOS CINÉTICOS DE LA HIDRATACIÓN DE LA ALITA (DRX, reflexión 1.76-1.78 A)

\begin{tabular}{|c|c|c|c|c|c|c|c|}
\hline \multirow{2}{*}{ Muestra } & \multicolumn{5}{|c|}{ Grado de reacción a } & \multicolumn{2}{|c|}{ Resultados cinéticos* } \\
\hline & 1d. & 3d. & 7d. & $28 d$. & 90d. & $K(10-3)$ & $\begin{array}{l}\text { Coeficiente de } \\
\text { correlación. }\end{array}$ \\
\hline $\mathbf{s}$ & ---- & 0,68 & 0,74 & 0,85 & 0,91 & 4.602 & 0,99 \\
\hline S1.1 & 0,56 & 0,60 & 0,63 & 0,86 & ----- & 6.600 & 0,99 \\
\hline S1.1 & ---- & 0,58 & 0,60 & 0,78 & ---- & 3.900 & 0,99 \\
\hline S1.3 & 0,08 & 0,10 & 0,42 & 0,72 & 0,82 & 2.000 & 0,94 \\
\hline S1.3A** & 0,32 & 0,60 & 0,75 & 0,82 & -.-- & 3.400 & 0,85 \\
\hline S1.4 & -.--- & 0,08 & 0,10 & 0,13 & 0,46 & $a<0,15$ & -..-- \\
\hline $\mathrm{S} 1.5$ & ---- & 0,08 & 0,09 & ---.- & 0,28 & $a<0,15$ & ----- \\
\hline s2 & $-\cdots$ & 0,12 & 0,32 & 0,78 & 0,83 & 2.165 & 0,87 \\
\hline s3 & ----- & 0,35 & 0,37 & 0,49 & 0,58 & 0.502 & 0,98 \\
\hline S4 & ----- & 0,38 & 0,60 & 0,76 & $\cdots$ & 4.232 & 0,99 \\
\hline S5 &.--- & 0,55 & 0,61 & 0,74 & ---- & 2.961 & 0,99 \\
\hline s6 & ----- & 0,80 & 0,84 & 0,92 & ---- & 5.860 & 0,99 \\
\hline s7 & ---- & 0,79 & 0,81 & 0,89 & ---- & 4.422 & 0,97 \\
\hline
\end{tabular}

* Modelo de Jander: $\left[1-(1-a)^{1 / 3}\right]^{2}$ vs. t., donde $K$ es la constante de velocidad de reacción

* * Cemento molido hasta superficie específica Blaine $3.642,3 \mathrm{~cm}^{2} / \mathrm{g}$.

TABLA 4

POROSIDAD A LOS 28 DIAS. PASTAS DE CEMENTO

\begin{tabular}{|c|c|c|c|c|c|c|}
\hline \multirow{2}{*}{ Muestra } & \multicolumn{3}{|c|}{ Volumen de poros $\left(\mathrm{cm}^{3} / \mathrm{g}\right)$} & \multicolumn{3}{|c|}{ Poros $(\%)$} \\
\hline & 94-250 $\mathrm{A}^{\circ}$ & 94-1071 $A^{\circ}(*)$ & $1071-75000 A^{\bullet}(* *)$ & Total & Grandes & Pequeños \\
\hline $\mathbf{s}$ & 0,0294 & 0,0671 & 0,0385 & 0,1056 & 36 & 64 \\
\hline s6 & 0,0366 & 0,0881 & 0,0334 & 0,1215 & 28 & 72 \\
\hline S5 & 0,1554 & 0,3191 & 0,1909 & 0,5100 & 37 & 63 \\
\hline S5.A & 0,0443 & 0,0672 & 0,0131 & 0,0803 & 16 & 83 \\
\hline S7 & 0,0388 & 0,0932 & 0,0596 & 0,1528 & 39 & 61 \\
\hline S4 & 0,0508 & 0,0698 & 0,0686 & 0,1384 & 50 & 50 \\
\hline
\end{tabular}

* Poros no dañinos $/ 3 /$

* * Poros dañinos $/ 3 /$ 
ligeramente la hidratación del cemento por su naturaleza orgánica (tabla 3), su principal acción es sobre la reología (tabla 2) y la estructura porosa de las pastas de cemento endurecida. Los resultados analizados tienen una relación directa con las resistencias mecánicas reportadas en la Fig. 1.

La influencia de los aditivos químicos en las propiedades reológicas de los morteros se observan en la tabla 6 . El A.S.P. logró una disminución en M3 de la relación $\mathrm{A} / \mathrm{C}$, con el conocido incremento de la pérdida de la laboriosidad (Fig. 5).

La adición de sacarosa permitió en M4 prolongar, en el tiempo, la consistencia y alcanzar mayor coherencia de la mezcla sin incremento de la relación $\mathrm{A} / \mathrm{C}$.
El efecto de ambos aditivos en el aumento de las propiedades mecánicas del mortero se observa en las figuras 2 y 3 . Las diferencias entre M2 y M4 hasta los 7 días no son significativas a pesar del efecto retardador de la sacarosa. Los resultados de permeabilidad (tabla 6) y de absorción (Fig. 4), confirman en M4 una estructura más compacta e impermeable.

\section{CONCLUSIONES}

Gracias a la incorporación de aditivos químicos, y a su acción vinculada con la fracción fina del árido $<45 \mu \mathrm{m}$, se mejoran las propiedades reológicas y físico-mecánicas de los morteros con áridos de tobas parcialmente zeolitizadas y esfactible su empleo en barcos de ferrocemento, piscinas, estanques, así como recubrimiento de edificios expuestos a la corrosión marítima.

TABLA 5

TECNOLOGIA DE PREPARACIÓN DEL MORTERO (MEZCLADORA DE GIRO PLANETARIO)

\begin{tabular}{||l|c|c|}
\hline \multicolumn{1}{|c|}{ Etapa } & Observación & Tiempo (minutos) \\
\hline Cemento + Agua (Pasta) & Homogeneizar & 1 \\
\hline Pasta + Aditivos químicos & Homogeneizar & 2 \\
\hline $\begin{array}{l}\text { Pasta + Árido * } \\
\text { Fracción 0-1 mm / 1-2,38 mm }\end{array}$ & $\begin{array}{l}\text { Homogeneizar } \\
\text { por separado }\end{array}$ & 2 \\
\hline
\end{tabular}

* Secos.

Tiempo $\min$

5

TABLA 6

CARACTERISTICAS DE LOS,MORTEROS

\begin{tabular}{|c|c|c|c|c|c|c|c|c|}
\hline \multirow{2}{*}{ Muestra } & \multicolumn{4}{|c|}{ Rolación gravimótrica } & \multirow{2}{*}{$\begin{array}{c}\text { Relación } \\
\text { A/C. }\end{array}$} & \multirow{2}{*}{$\begin{array}{l}\text { Fluidez } \\
\text { (mm) }\end{array}$} & \multirow{2}{*}{$\begin{array}{l}\text { Pórdida de } \\
\text { laborabilidad } \\
\text { a } 45 \text { min. }(\%)\end{array}$} & \multirow{2}{*}{$\begin{array}{c}\text { Permeabilidad } \\
\text { JIS A. } 6203 \\
\text { (\% P/P) }\end{array}$} \\
\hline & Comento & Árido & A.S.P.(\%) & A.R. $(\%)$ & & & & \\
\hline M1 & 1 & $2 * *$ & $-\cdots$ & ---- & 0,50 & 100 & $\cdots$ & --.-- \\
\hline M2 & 1 & $1,6 * * *$ & $-\cdots$ & $-\cdots$ & 0,80 & 110 & $\cdots$ & 25 \\
\hline M3 & 1 & $1,6 * * *$ & 0,600 & ---- & 0,62 & 120 & 41 & 17 \\
\hline M4 & 1 & $1,6 * * *$ & 0,600 & 0,400 & 0,55 & 120 & 19 & 10 \\
\hline
\end{tabular}

* Norma Industrial Japonesa.

* * Árido silíceo cuarzoso.

* * Árido de toba zeolitizada. 


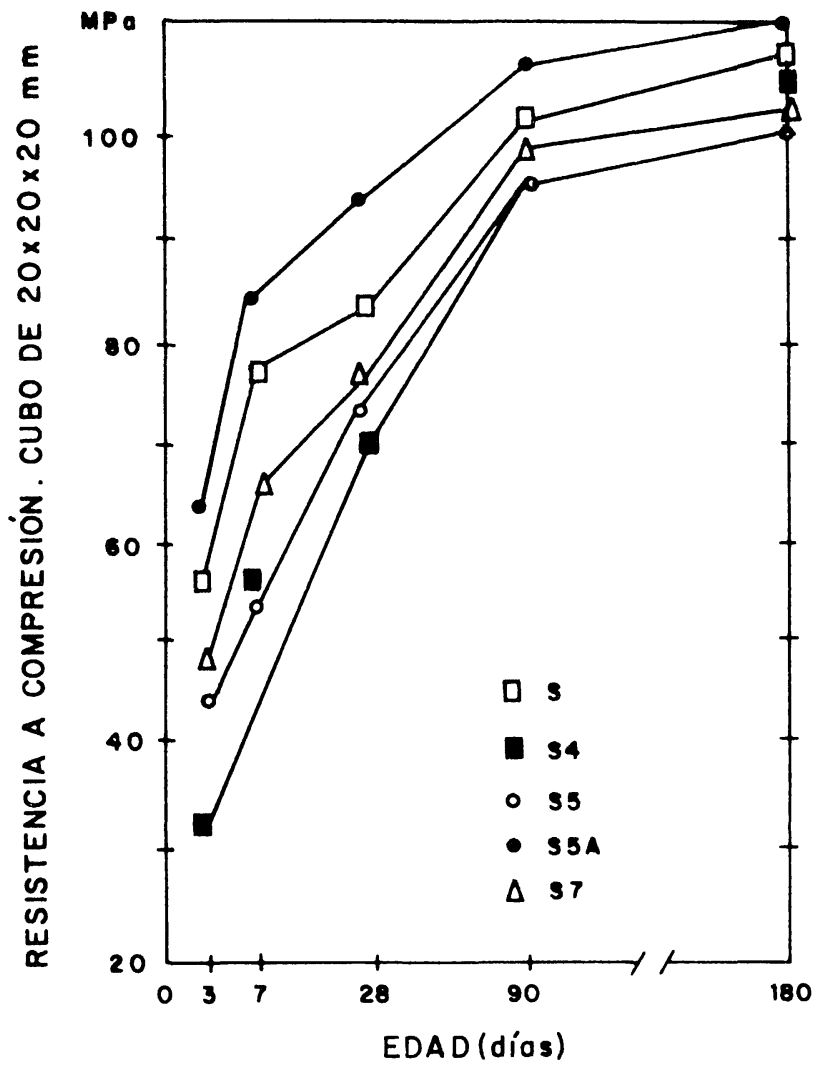

Fig. 1.- Influencia del tiempo en la resistencia a compresión de las pastas con aditivos químico-mineral.

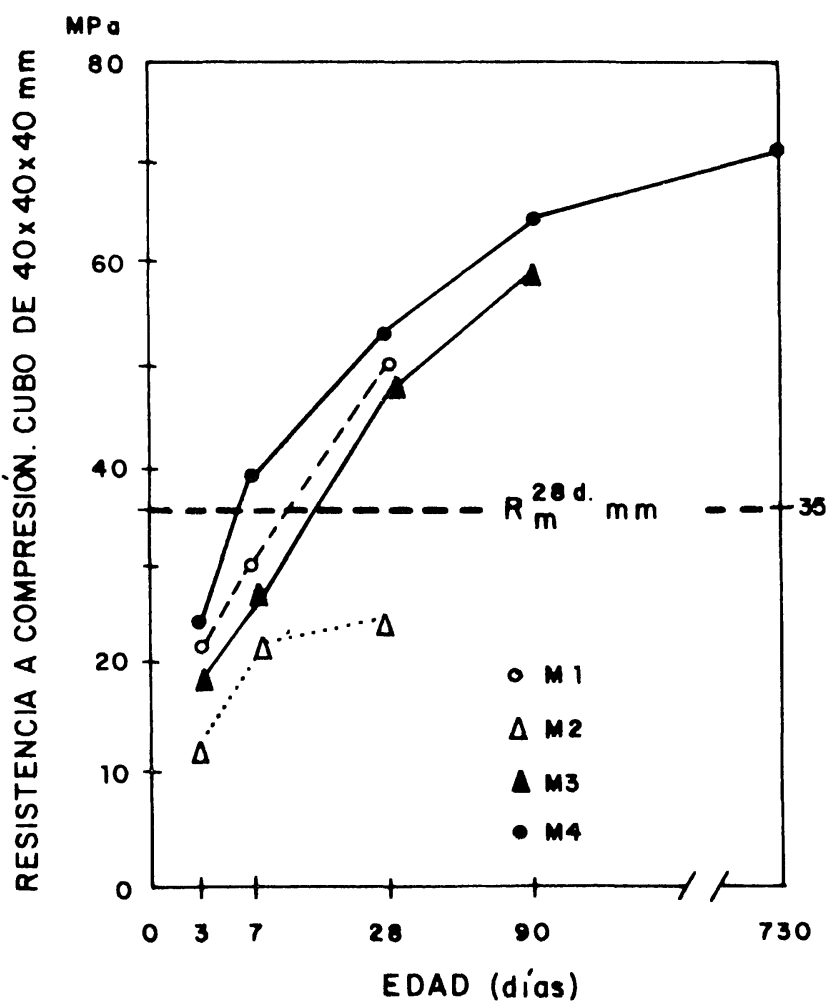

Fig. 3.- Influencia del tiempo en la resistencia a compresión de morteros ligeros con aditivos químico-mineral.

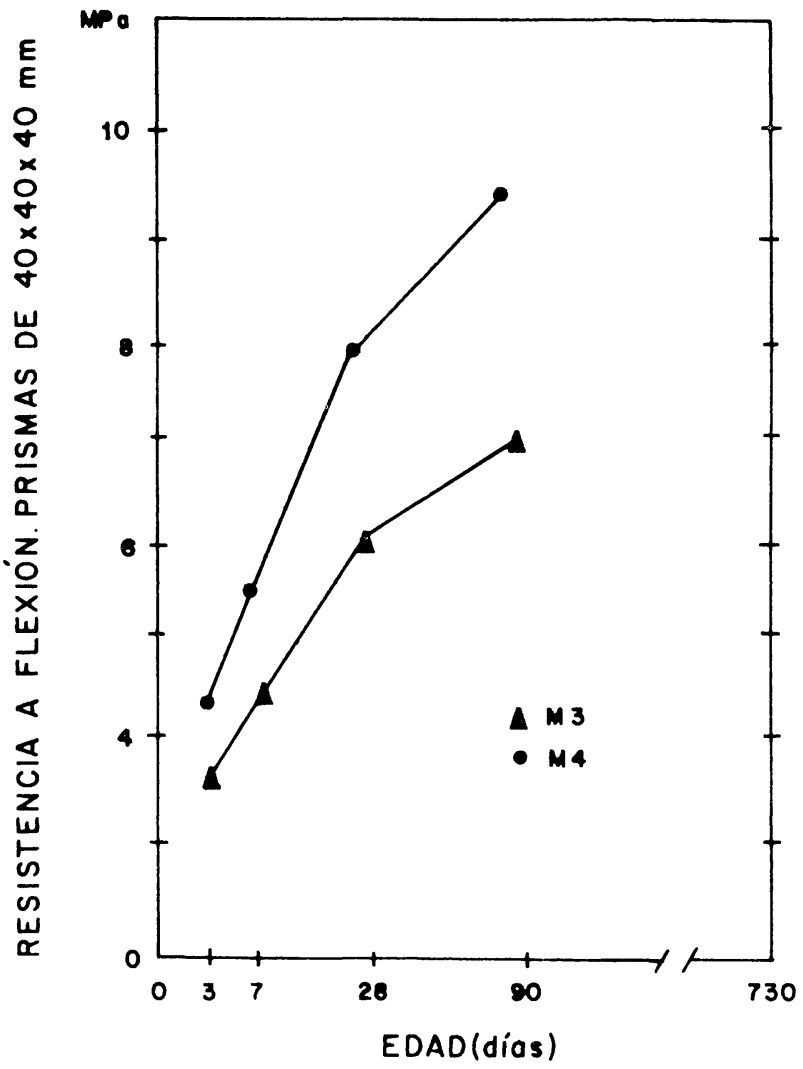

Fig. 2.- Influencia del tiempo en la resistencia a la flexión de morteros con aditivos químico-mineral.

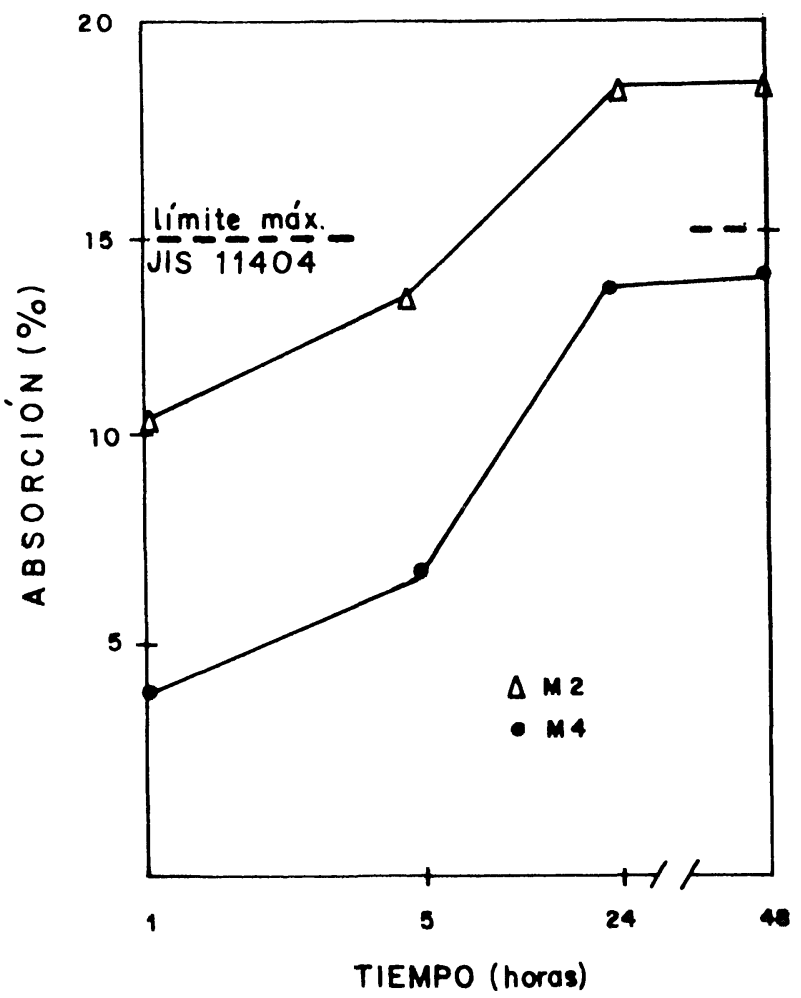

Fig. 4.- Influencia del tiempo en el \% absorción de los morteros. 


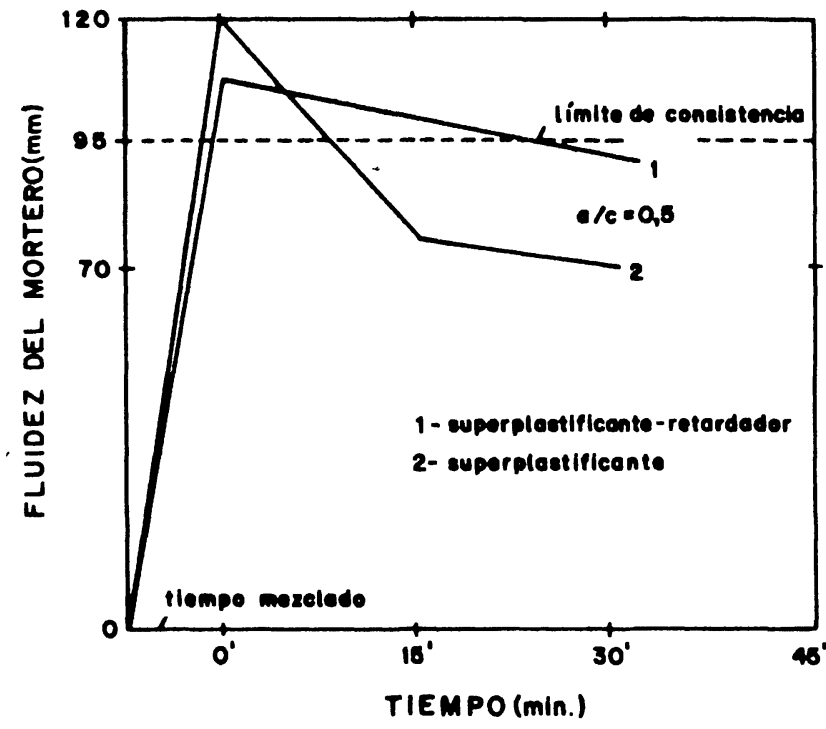

Fig. 5.- Influencia de los aditivos químicos en la pérdida de consistencia de morteros ligeros con zeolitas naturales sílicoactiva.

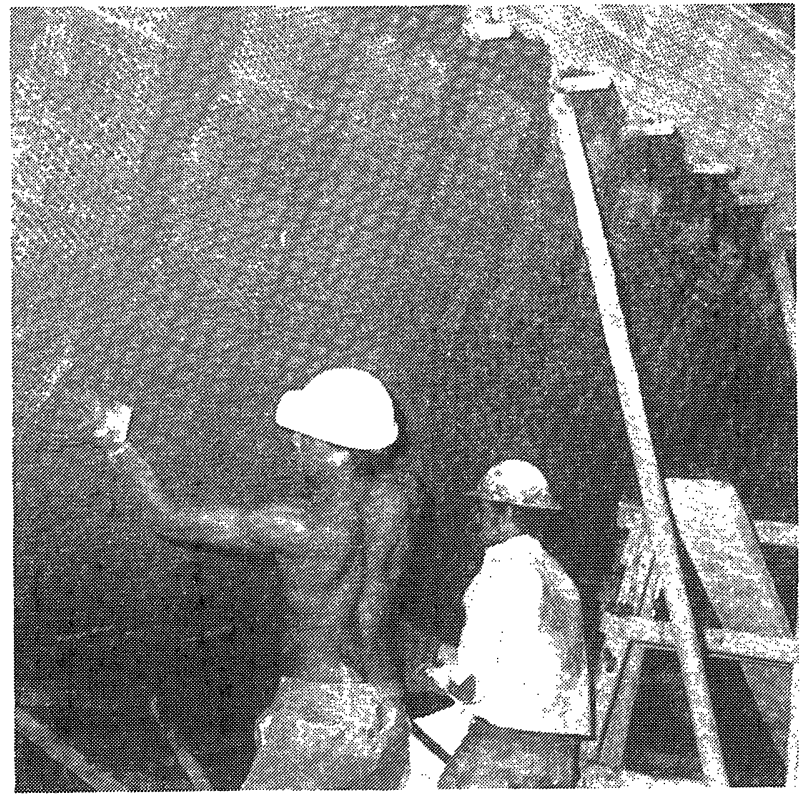

Aplicación de morteros ligeros con áridos de tobas parcialmente zeolitizadas en barcos de ferrocemento.

\section{BIBLIOGRAFÍA}

(1) KANTRO, D.L. ASTM C-1. Symposium on Non-Standard Methods for Cement Testing. California, USA. 1979.

(2) Sotolongo, R, GALloso, R., GALVEZ, M. Revista Materiales de Construcción. Vol. 43. No. 230. España. 1993.

(3) MOR, A., MEHTA, P.K. Cement and Concrete Research. Vol. 14. pp. 754-756. USA. 1984.

(4) GAYOSO, R., GIL, C. "Evaluación de yacimientos de tobas destinadas a la producción de áridos ligeros", Informe CTDMC. Cuba. 1991.

(5) GENER, M. Boletín No. 3. CIEC. Cuba. 1979.

(6) BALMAYOR, M., SOTOLONGO, R., MONTALVO, S. Memorias QUIMINDUSTRIA'93. VII Seminario del CIQ. Cuba. 1993.

(7) DUQUE, G.L., LLOPIZ, J., RUBIO, E. Revista Materiales de Construcción. Vol. 39. No. 312. España. 1989.

(8) MEHTA, P.K. D1. 1988. Durability of Concrete. International Seminar. Swedish. 1989. 